\title{
Mid-infrared images of the star forming region GGD 14 (IRAS 06084-0611)^
}

\author{
P. Persi ${ }^{1}$ and M. Tapia ${ }^{2}$ \\ ${ }^{1}$ Istituto Astrofisica Spaziale e Fisica Cosmica, CNR, Via del Fosso del Cavaliere, 00133 Roma, Italy \\ 2 Instituto de Astronomía, UNAM, Apartado Postal 877, Ensenada, Baja California, CP 22830, Mexico
}

Received 4 March 2003 / Accepted 7 May 2003

\begin{abstract}
Mid-infrared images at 8.7, 9.7 and $12.5 \mu \mathrm{m}$ are presented of the star forming region GGD 14 associated with the source IRAS 06084-0611. In an area of $\sim 30^{\prime \prime}$ around the IRAS position, two mid-infrared sources were found. One was identified with the cometary compact HII region VLA 1 and the second with the faint and unresolved radio continuum source VLA 4. The mid-infrared morphology of VLA 1 is very similar to that of the radio continuum suggesting a direct interaction between the ionized gas and the dust. The infrared source associated with VLA 4 shows a large infrared excess and its derived infrared luminosity is $L_{1-20 \mu \mathrm{m}}=350 L_{\odot}$, indicating the presence of a young star later than B3. In addition, its spectrum between 2.47 and $11.62 \mu \mathrm{m}$ observed by ISOPHOT shows the presence of infrared emission bands at 3.3, 6.2, 7.7, 8.6 and $11.2 \mu \mathrm{m}$ with relative intensity ratios typical of compact HII regions. Finally, the radio continuum source VLA 2, undetected on our mid-infrared images, shows an infrared luminosity of $\sim 8.5 L_{\odot}$ confirming its nature of an embedded T-Tauri star.
\end{abstract}

Key words. stars: formation - infrared: stars

\section{Introduction}

The red nebulous object GGD 14 discovered by Gyulbudaghian et al. (1978) is embedded in the Monoceros molecular cloud at a distance of $\sim 1 \mathrm{kpc}$ (Racine \& van den Bergh 1970; Rodríguez et al. 1980). This object is within an active star forming region as shown by the presence of a well-known cometary HII region (VLA 1) ionized by a B0.5 zero-age main-sequence (ZAMS) star (Tofani et al. 1995; Gómez et al. 1998; Gómez et al. 2000) and associated with the IRAS source 06084-0611. No point-like near-infrared source at a level of $K \geq 14$ has been detected at the central position of the cometary HII region, although maps at 20, 50, 100 and $800 \mu \mathrm{m}$ obtained by Harvey et al. (1985) and Little et al. (1990) show strong emission centered on this source. This implies a visual extinction larger than 55 mag in the direction of the HII region.

A young cluster was found by means of radio and infrared observations in a region of $30^{\prime \prime}$ around the compact cometary HII region (Gómez et al. 2000; Harvey et al. 1985). Most of the members of the cluster (at least 8 components) could be low-mass pre-main-sequence stars as suggested by recent radio continuum observations at 3.6 and 6 cm (Gómez et al. 2002).

Send offprint requests to: P. Persi, e-mail: persi@rm.iasf.cnr.it

* Based on observations collected at the Observatorio Astronómico Nacional, San Pedro Mártir, B.C, Mexico, and ISOPHOT data from the ISO Data Archive (TDT No.70901306).
Another active center of star formation in GGD 14 is located approximately $30^{\prime \prime} \mathrm{NE}$ of the compact HII region close to the center of a CO bipolar outflow (Rodriguez et al. 1982), where a water maser source (Rodriguez et al. 1980; Tofani et al. 1995) and a radio continuum source (VLA 7) with an associated infrared source (Gómez et al. 2002) are also located. VLA 7 is probably the powering source of the molecular outflow.

Spectrophotometry between 2.47 and $11.62 \mu \mathrm{m}$ has been obtained with ISOPHOT (PHT-S, Lemke et al. 1996) around the IRAS position. In this work we make use of ISOPHOT data of this source from the ISO Data Archive (TDT No. 70901306).

In this paper we report the results of mid-infrared images centered on the IRAS position and we discuss the spectral energy distribution (SED) of the detected sources together with the ISOPHOT spectrophotometry.

\section{Observations}

\subsection{Mid-infrared images}

The narrow-band images at 8.7, 9.7 and $12.5 \mu \mathrm{m}$ centered at the position of the IRAS source 06084-0611 in GGD 14 were taken the night of November 92001 with the mid-infrared camera CID (Salas et al. 2003) on the $2.1 \mathrm{~m}$ telescope of the Observatorio Astronómico Nacional at San Pedro Mártir, Baja California, Mexico. This camera is equipped with a Rockwell $128 \times 128$ pixel Si:As BIB detector array and delivers an effective scale of $0.55^{\prime \prime} /$ pix. The images were taken in the standard 

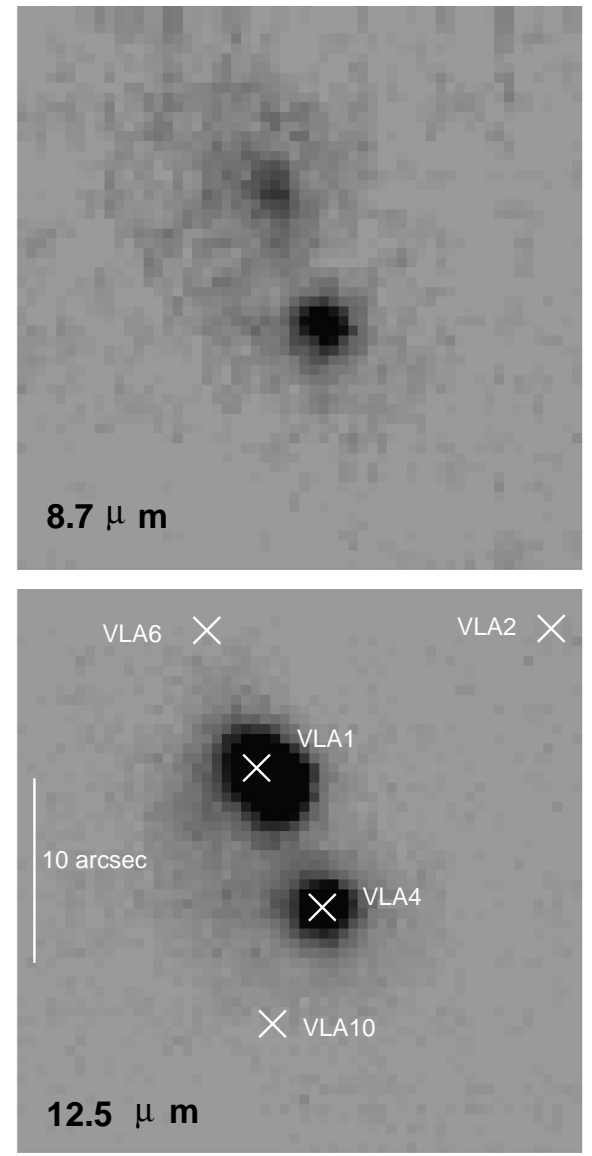


Fig. 1. Mid-infrared images of GGD 14 (IRAS 06084-0611). For comparison, the $2.2 \mu$ m image by Kodapp (1994) is also shown at the same scale. The positions of the continuum radio sources found by Gómez et al. (2002), are marked on the $12.5 \mu \mathrm{m}$ image. North is the top and east to the left.

chop-nodding technique to remove the sky and telescope emission background. The standard stars $\alpha$ Tau and $\alpha$ Aur were observed before and after the source at similar air-mass for flux calibration. The images of these stars were also used to estimate the point-spread function at each wavelength. The mean PSF was $\sim 1.5^{\prime \prime}(F W H M)$, close to the diffraction limit. The on-source integration times were $570 \mathrm{~s}$ at $8.7 \mu \mathrm{m}$, and $720 \mathrm{~s}$ at 9.7 and $12.5 \mu \mathrm{m}$. The derived detection limits are $\sim 0.2 \mathrm{Jy}$, $0.5 \mathrm{Jy}$ and $0.3 \mathrm{Jy}(1 \sigma)$ respectively.

Two distinct bright mid-infrared sources separated by $0.2^{\mathrm{s}}$ in RA and 7.3" in Dec are present in all mid-infrared images. We identify these two sources with the cometary HII region VLA 1, and with the faint compact radio continuum source VLA 4 (see Fig. 1). These are also sources Irs 4 and Irs 2 observed by Harvey et al. (1985) and their $20 \mu \mathrm{m}$ map of Fig. 4 is very similar to our $12.5 \mu \mathrm{m}$ image. The mid-infrared emission from the other three radio continuum sources VLA 2, VLA 6, and VLA 10 (Gómez et al. 2002) are below our detection limits, although included in the observed field. With the exception of VLA 1, all the radio continuum sources have associated a near-infrared source as seen in Hodapp's (1994) $K_{\mathrm{s}}$ image also displayed in Fig. 1. The mid-infrared source associated with the cometary HII region is slightly elongated in the NE-SW direction, while the mid-infrared emission from VLA 4 appears point-like.
The photometry of the two mid-infrared sources was performed with the DAOPHOT package within IRAF (Stetson 1987). The flux densities, the $1 \sigma$ statistical errors, and the aperture used for the photometry of the two sources, are given in Table 1 . The IRAS flux observed at $12 \mu \mathrm{m}$ of $27.1 \mathrm{Jy}$ probably include both sources, VLA 1 and VLA 4.

\subsection{ISOPHOT spectrophotometry}

Spectrophotometry between 2.47 and $11.62 \mu \mathrm{m}$ towards IRAS 06084-0611 was obtained with PHT-S (PHT40) on 25 October 1997. The coordinates of the center of the $24{ }^{\prime \prime} \times 24^{\prime \prime}$ aperture of PHT-S on ISO are recorded in the observing log, namely $\alpha_{2000}=6^{\mathrm{h}} 10^{\mathrm{m}} 50.2^{\mathrm{s}} ; \delta_{2000}=6^{\circ} 12^{\prime} 1.1^{\prime \prime}$ with a probable error of $2-3^{\prime \prime}$ (ISO Handbook, Vol. I). This nominal position is $2^{\prime \prime}$ west and $4^{\prime \prime}$ south of the VLA position of source VLA 4 (Irs 2) and 6" west and 11" south of source VLA 1 (Irs 4).

We obtained the PHOT-S calibrated scientific data from the ESO Data Archive (TDT No. 70901306). Figure 2 illustrates the spectrum with a total integration time of $620 \mathrm{~s}$. In the figure, our flux densities for source VLA 4 (open circles) are also reported. The main uncertainty in this observation is the extent to which the flux from the northernmost infrared source (VLA 1) is also included in the aperture, as this is nominally 
Table 1. Mid infrared photometry of VLA 1 and VLA 4.

\begin{tabular}{lllll}
\hline \hline Source & $F_{8.7}$ & $F_{9.7}$ & $F_{12.5}$ & Ap. \\
& $\mathrm{Jy}$ & $\mathrm{Jy}$ & $\mathrm{Jy}$ & ${ }^{\prime}$ \\
\hline VLA 1(Irs4) & $1.5(0.2)$ & $9.7(0.7)$ & $14.9(0.7)$ & 4.4 \\
VLA 4(Irs2) & $2.2(0.2)$ & $4.6(0.5)$ & $7.3(0.6)$ & 3.3 \\
\hline
\end{tabular}

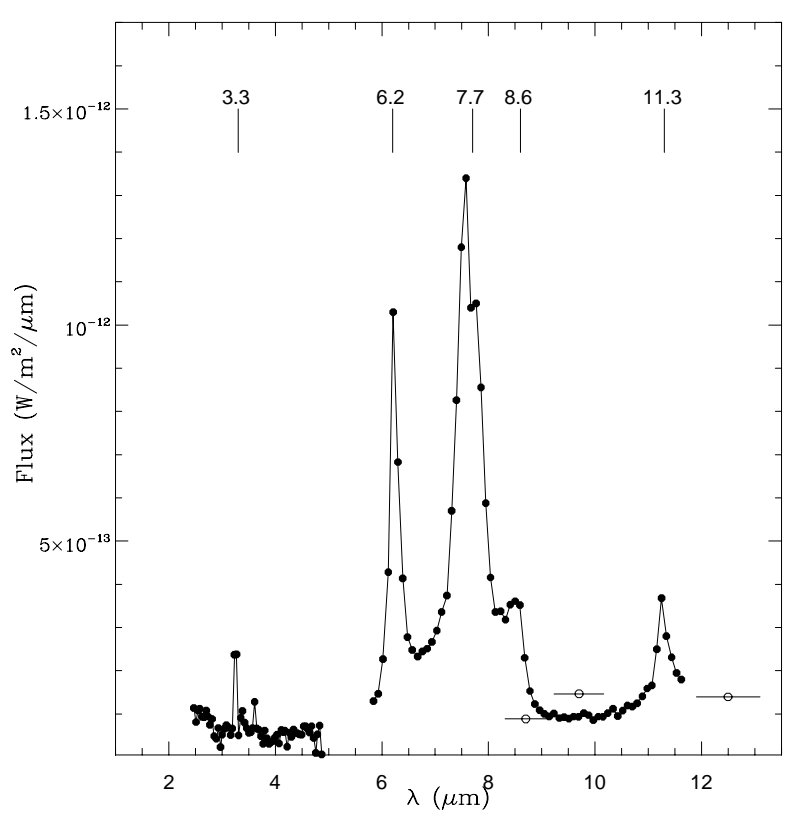

Fig. 2. The PHT-S spectrum towards the IRAS source 06084-0611. The open circles represents our observed flux densities of the source VLA 4 (Irs2). The bars show the widths of the mid-infrared filters.

Table 2. Measured line fluxes of the IEB.

\begin{tabular}{cc}
\hline \hline IEB $(\mu \mathrm{m})$ & Line Flux $\left(10^{-14} \mathrm{~W} \mathrm{~m}^{-2}\right)$ \\
\hline 3.3 & 1.7 \\
6.2 & 18.5 \\
"7.7” & 64.7 \\
8.6 & 10.6 \\
11.2 & 8.4 \\
\hline
\end{tabular}

located close or at the edge of the square aperture of the instrument. Another serious caveat that has to be taken into account is that the PHT-S spectrophotometry calibrations are accurate only for sources within $\pm 3^{\prime \prime}$ of the center of the aperture (ISO Handbook, Vol. IV).

The spectrum is dominated by the infrared emission bands (IEB) at 3.3, 6.2, “7.7", 8.6 and $11.2 \mu \mathrm{m}$ attributed to polycyclic aromatic hydrocarbons (PAHs). In particular, the socalled "7.7" $\mu \mathrm{m}$ band appears to be composed of two emission bands at 7.6 and $7.8 \mu \mathrm{m}$. This double peak is also seen in other compact HII regions (Roelfsema et al. 1996) and other types of sources. The measured line fluxes of these bands are reported in Table 2. Considering the arguments given at the begining of this section, we estimate that the fluxes reported in Fig. 2 and Table 2 are accurate only to around 25\% (see also Mattila et al. 1996).
As mentioned before, we find enough evidence to believe that the ISO spectrum is dominated by source VLA 4 (Irs 2): it is close to the center of the PHT-S aperture in contrast to VLA 1 which lies close to the edge of the PHT-S aperture. Apart from the fact that VLA 1 is extended and may be partially out of the aperture, this ISO instrument exhibits a steep beam profile (ISO Handbook, Vol. IV) which affects very strongly the measured flux away from the center of the field. We cannot exclude, though, that VLA 1 may contribute to a small fraction of the measured flux. It should be mentioned that the fluxes of VLA 4 from our images are consistent with those implied by the satellite spectrum.

\section{Discussion}

Figure 3 illustrates the comparison between our $12.5 \mu \mathrm{m}$ contour map and the $3.6 \mathrm{~cm}$ contour map from Gómez et al. (2002). Two of the five radio sources present in our observed field (VLA 1 and VLA 4), were detected in the midinfrared. VLA 2, 4 and 10 are coincident with the near-infrared sources found in the Two Micron All Sky Survey (2MASS) (see Gómez et al. 2002) and in the $K$-band image of Hodapp (1994) (Fig. 1). Using the 2MASS photometry, we derived their positions in the $J-H$ vs. $H-K$ diagram, which is presented in Fig. 4. The analysis of this diagram indicates that VLA 2 is a very highly reddened star with a small near-infrared excess, while the location of VLA 10 suggests this source is a latetype star $\left(A_{V}=5-6\right)$ with no near-infrared excess. In contrast, a significant near-infrared excess is observed towards VLA 4. Gómez et al. (2002) proposed a possible T-Tauri nature for these sources to explain their observed negative radio spectral index and radio variability.

In the following sections we discuss individually the nature of the three radio sources VLA 1, VLA 4 and VLA 2.

\subsection{VLA 1 (Irs 4)}

The compact cometary HII region VLA 1 shows a similar morphology and extension in the mid-infrared and in the radio continuum with a sharp edge on the western side (Fig. 3). This suggests a direct interaction between the ionized gas and the dust cloud.

Combining our mid-infrared flux densities of Table 1, the narrow-band photometry around 10 and $20 \mu \mathrm{m}$ of Harvey et al. (1985), the $K_{\mathrm{s}}$-band upper limit taken from 2MASS, and the sub-millimeter and millimeter observations at 450, 800 and $1100 \mu \mathrm{m}$ with a $15^{\prime \prime}$ beam from Little et al. (1990), we obtain the spectral energy distribution (SED) of VLA 1 shown in Fig. 5. The IRAS flux densities reported in the SED include the emission of both sources, VLA 1 and VLA 4, as discussed in Sect. 2.1. Therefore, the derived luminosity of $\sim 9.6 \times 10^{3} L_{\odot}$, obtained including the far-infrared observations (Gómez et al. 2002) is for the two sources.

Finally, the SED of VLA 1 appears very steep with the infrared spectral index

$\alpha_{\mathrm{IR}}=\mathrm{d} \log \lambda F_{\lambda} / \mathrm{d} \log \lambda \geq 4.5$

measured between 2.2 and $12.5 \mu \mathrm{m}$. 

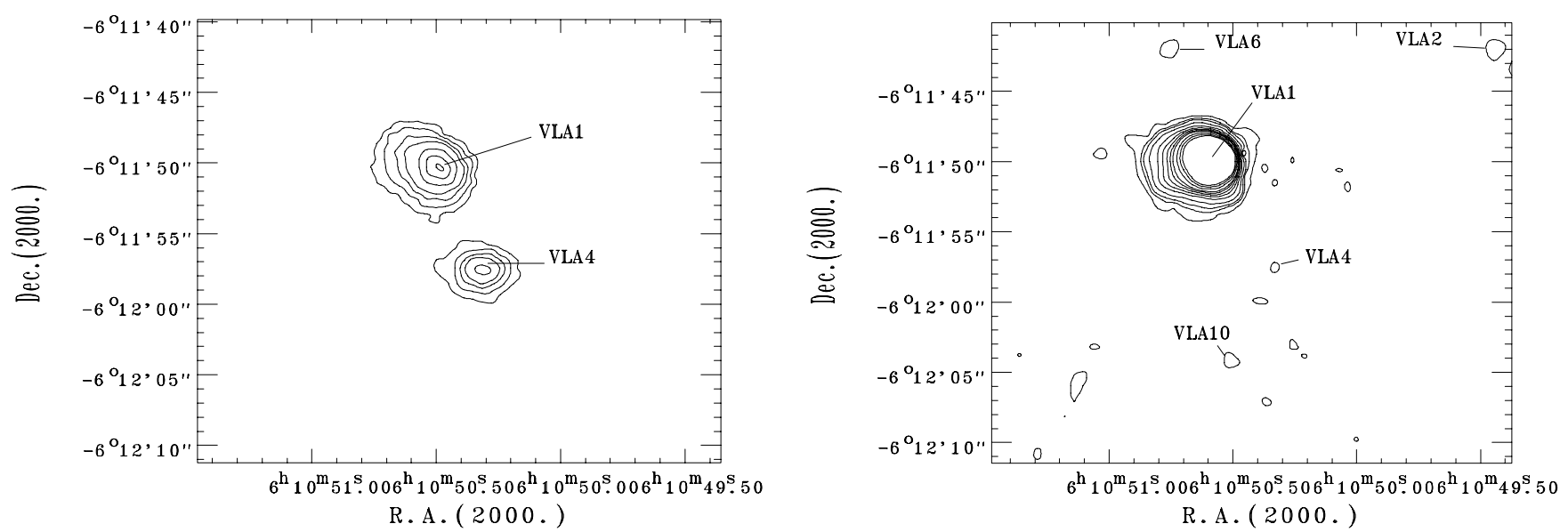

Fig. 3. Contour maps at $12.5 \mu \mathrm{m}$ (left panel) and at $3.6 \mathrm{~cm}$ (right panel) from (Gómez et al. 2002) of the star forming region GGD 14 around IRAS 06084-0611. The contour levels in the $12.5 \mu \mathrm{m}$ map are: 0.36, 0.45, 0.54, 0.62, 0.71, 0.80 and $0.89 \mathrm{Jy} / \mathrm{arcsec}^{2}$.

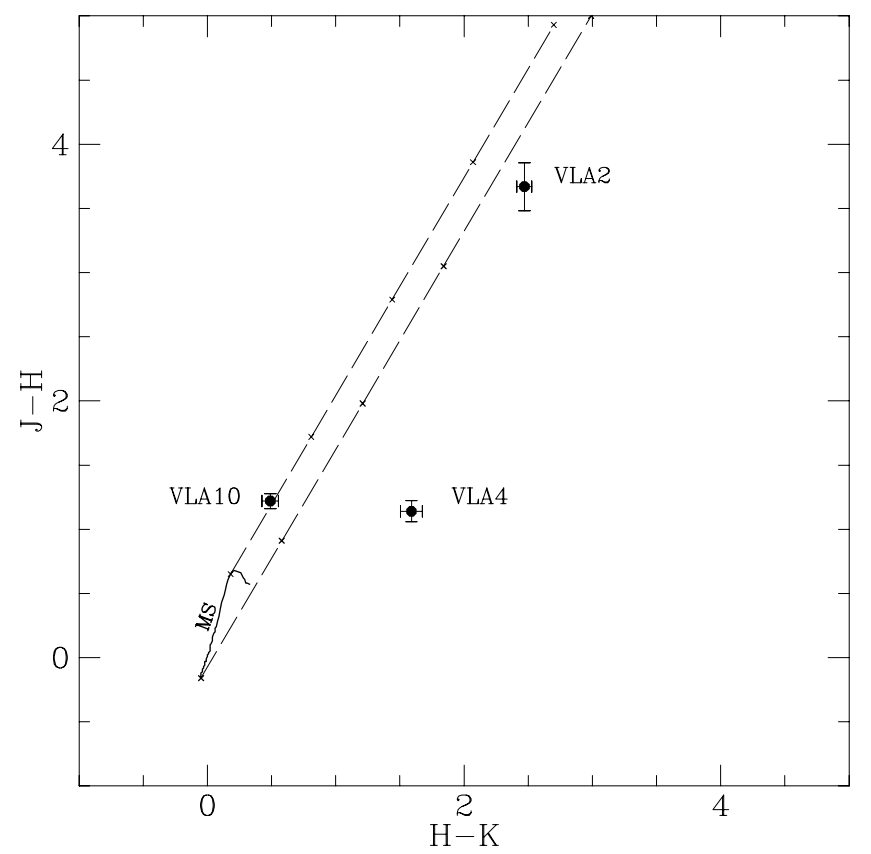

Fig. 4. $J-H$ vs. $H-K$ diagram of the VLA sources in the GGD 14 region. The continuous line marks the locus of the main sequence stars from Koornneef (1983). The two parallel lines represent the standard reddening vectors for late and early-type stars.

\subsection{VLA 4 (Irs 2)}

The mid-infrared emission of VLA 4 appears point-like on our images. The SED of this source obtained from the near and mid-infrared photometry of Harvey et al. (1985) and our flux densities of Table 1 taken with a similar aperture is flatter than that of VLA 1 , having a spectral index $\alpha($ IR $)=2.2$ (left panel of Fig. 6). Integrating the infrared energy distribution and assuming a distance of $1 \mathrm{kpc}$, we obtain a luminosity between 1 and $20 \mu \mathrm{m} L_{1-20 \mu \mathrm{m}}=350 L_{\odot}$. This value is consistent with the presence of a very young star somewhat later than B3. In addition, the measured intensity ratios $I(3.3) / I(11.2)=0.20$, and $I(6.2) / I(7.6)=0.29$ of the PAH bands present in the ISOPHOT



Fig. 5. Spectral energy distribution of VLA 1. The symbols are: open circles from Harvey et al. (1985), open squares from Little et al. (1990) with a $15^{\prime \prime}$ beam, filled circles from this work. The $K$ upper limit is taken from 2MASS.

spectrum (Fig. 2), are very similar to a number of Herbig Ae/Be stars and compact HII regions. This young object is probably a Herbig Ae/Be star.

\subsection{VLA 2 (Irs 6)}

VLA 2 is a very faint and unresolved radio source $\left(\leq 0.3^{\prime \prime}\right)$ detected by Gómez et al. (2000) and associated with source Irs 6 of Harvey et al. (1985). Its spectral energy distribution (right panel of Fig. 6) is obtained from the near-infrared photometry of 2MASS and from the photometry up to $20 \mu \mathrm{m}$ of 

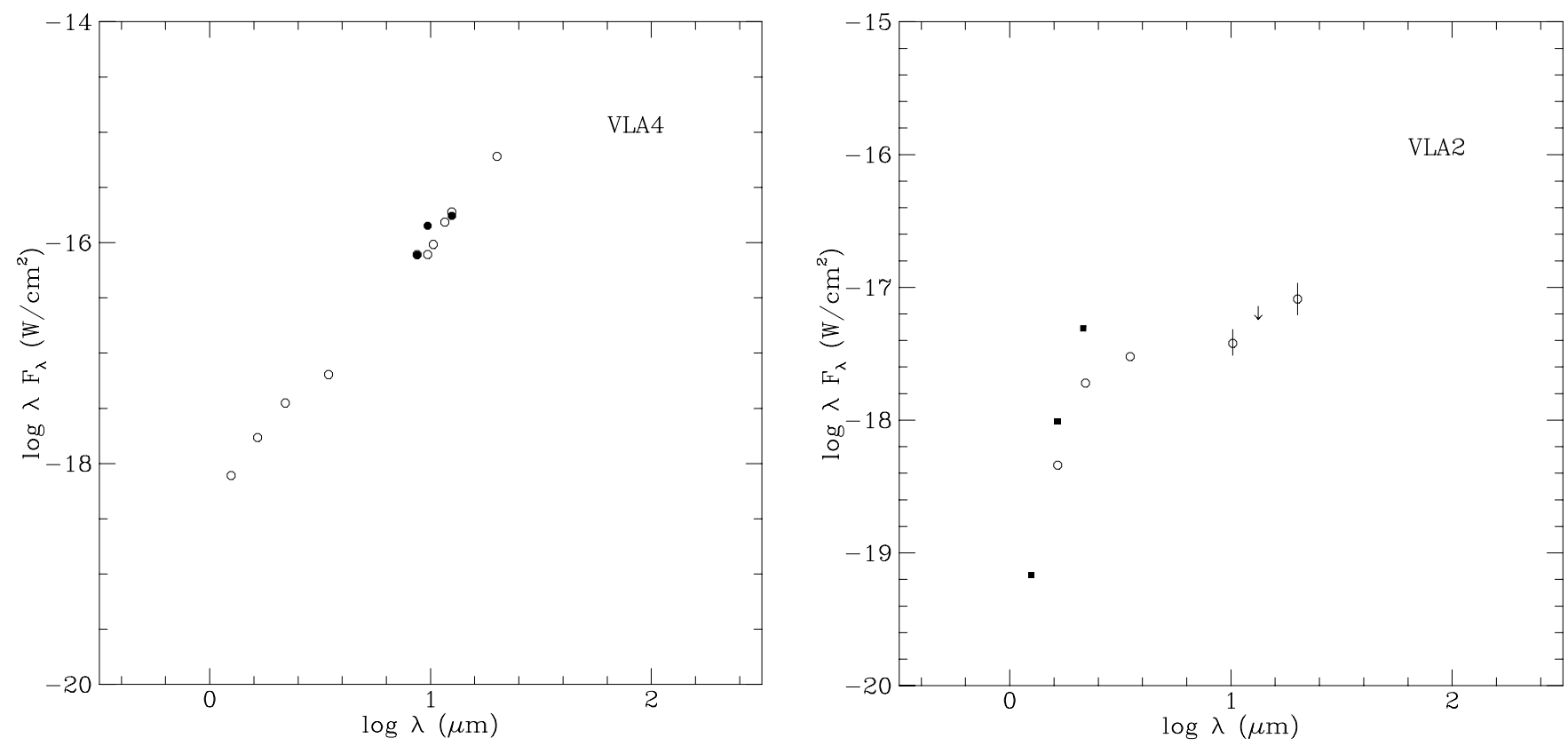

Fig. 6. Left panel: SED of VLA 4. The symbols are: open circles from Harvey et al. (1985) and filled circles from this work. Right panel: SED of VLA 2. The symbols are: open circles from Harvey et al. (1985) and filled squared from 2MASS.

Harvey et al. (1985). Our upper limit at $12.5 \mu \mathrm{m}$ is also reported in the SED.

The two sets of near infrared observations (2MASS and Harvey et al. 1985) suggest that this source is variable as shown in the right panel of Fig. 6. This fact, together with the estimated $1-20 \mu \mathrm{m}$ luminosity of $\sim 8.5 L_{\odot}$, indicate that VLA 2 is an embedded T-Tauri star, in agreement with the conclusion given for this object by Gómez et al. (2002).

\section{Conclusions}

From the analysis of the images at 8.7, 9.7 and $12.5 \mu \mathrm{m}$ of a region of size $\sim 30^{\prime \prime}$ around GGD 14 around IRAS 06084-0611) we reached the following conclusions:

1) Two mid-infrared sources associated with the cometary compact HII region VLA 1 and with the faint radio continuum source VLA 4 have been found. The two sources contribute to the observed IRAS fluxes. VLA 1 shows a diffuse mid-infrared emission very similar to that of radio continuum at $3.6 \mathrm{~cm}$ (Fig. 3). Its energy distribution is very steep with the infrared spectral index $\alpha($ IR $) \geq 4.5$. The bolometric luminosity of this source is $<9.6 \times 10^{3} L_{\odot}$.

2) The mid-infrared source associated with VLA 4 shows an infrared excess and its derived infrared luminosity of $L_{1-20 \mu \mathrm{m}}=350 L_{\odot}$ is consistent with the presence of a young embedded star of spectral type somewhat later than B3. Infrare emission bands at 3.3, 6.2, "7.7", 8.6 and $11.2 \mu \mathrm{m}$ have been observed by ISOPHOT towards this source (Fig. 2). The double peak of the "7.7" $\mu \mathrm{m}$ band and the relative intensity ratios are typical of compact HII regions.

3 ) The three other radio continuum sources present in our region, VLA 2, 6 and 10, and classified as possible T-Tauri stars by Gómez et al. (2002), were not detected in the mid-infrared with our sensitivity. Particularly interesting is VLA 2 that is associated with the infrared source Irs 6 of Harvey et al. (1985). This very red source has a luminosity between $1-20 \mu \mathrm{m}$ of $\sim 8.5 L_{\odot}$ and shows variability in the near-infrared. This confirms the presence of an embedded T Tauri star.

With our available data, it is impossible to classify for sources VLA 6 and VLA 10.

In summary, we can confirm that the star forming region GGD 14 is a very young complex where stars of different masses ranging from high mass (VLA 1) to intermediate (VLA 4) to low-mass star (VLA 2) are detected in the radio and infrared.

Acknowledgements. Part of this work was supported by a CNR(Italy)Conacyt(Mexico) bilateral agreement. MT also acknowledges grants from Conacyt and DGAPA-UNAM (IN-105400). We would like to thank Yolanda Gómez for the $3.6 \mathrm{~cm}$ image. This publication makes use of data products from the Two Micron All Sky Survey, which is a joint project of the University of Massachusetts and the Infrared Processing and Analysis Center/California institute of Technology, funded by the National Aeronautics and Space Administration and the National Science Foundation.

\section{References}

Gómez, Y., Lebron, M., Rodriguez, L. F., et al. 1998, ApJ, 503, 297

Gómez, Y., Rodriguez, L. F., \& Garay, G. 2000, ApJ, 531, 861

Gómez, Y., Rodriguez, L. F., \& Garay, G. 2002, ApJ, 571, 901

Gyulbudaghian, A. L., Glushkov, Yu. I., \& Denisyuk, K. E. 1978, ApJ, 224, L137

Harvey, P., Wilking, B. A., Joy, M., \& Lester, D. F. 1985, ApJ, 288, 725

Hodapp, K. W. 1994, ApJS, 94, 615

Koornneef, J. 1983, A\&A, 128, 84

Lemke, D., Klaaas, U., Abolins, J., et al. 1996, A\&A, 315, L65 
Little, L. T., Heaton, B. D., \& Dent, W. R. F. 1990, A\&A, 232, 173

Mattila, K., Lemke, D., Haikaøa, L. K., et al. 1996, A\&A, 315, L353

Racine, R., \& van den Bergh, S. 1970, in IAU Symp., 38, The Spiral Structure of our Galaxy, ed. W. Becker, \& G. Contopoulos (Dordrecht: Reidel), 219

Rodriguez, L. F., Moran, J. M., Ho, P. T. P., \& Gottlieb, W. 1980, ApJ, 235,845

Rodriguez, L. F., Carrall, P., Ho, P. T. P., \& Moran, J. M. 1982, ApJ, 260,635
Roelfsema, P. R., Cox, P., Tielens, A. G. G. M., et al. 1996, A\&A, 315, L289

Salas, L., Gutierrez, L., Tapia, M., et al. 2003, in Instrument Design and Performance for Optical/Infrared Ground-Based Telescopes, ed. I. Mesanori, \& A. F. M. Moorwood, SPIE Proc., 4841

Stetson, P. B. 1987, PASP, 99, 191

Tofani, G., Felli, M., Taylor, G. B., \& Hunter, T. R. 1995, A\&AS, 112, 299 